\title{
Real-time chest-wall-motion tracking by a single optical fibre grating: a prospective method for ventilator triggering
}

\author{
M D Ivanović ${ }^{1}$, J Petrovic ${ }^{1}$, A Savić ${ }^{2}$, G Gligorić ${ }^{1}$, M Miletić ${ }^{1}$, M Vukčević ${ }^{3}$, B Bojović ${ }^{1}$, \\ Lj Hadžievski ${ }^{1}$, T Allsop ${ }^{4}$, and D J Webb ${ }^{4}$ \\ ${ }^{1}$ Vinča Institute of Nuclear Sciences, University of Belgrade, Mike Petrovića Alasa 12-14, 11001 \\ Belgrade, Serbia \\ ${ }^{2}$ School of Electrical Engineering, University of Belgrade, Bulevar kralja Aleksandra 73, 11000 \\ Belgrade, Serbia \\ ${ }^{3}$ School of Medicine, University of Belgrade, Dr Subotića 8, 11000 Belgrade, Serbia \\ ${ }^{4}$ Aston Institute of Photonic Technologies, Aston Triangle, B4 7ET Birmingham, UK \\ email: marijap@ vin.bg.ac.rs
}

\begin{abstract}
:
Objective: The ventilators involved in non-invasive mechanical ventilation commonly provide ventilator support via a facemask. The interface of the mask with a patient promotes air leaks that cause errors in the feedback information provided by a pneumatic sensor and hence patient-ventilator asynchrony with multiple negative consequences. Our objective is to test the possibility of using chest-wall motion measured by an optical fibre-grating sensor as a more accurate non-invasive ventilator triggering mechanism.
\end{abstract}

Approach: The basic premise of our approach is that the measurement accuracy can be improved by using a triggering signal that precedes pneumatic triggering in the neuroventilatory coupling sequence. We propose a technique that uses the measurement of chest-wall curvature by a long-period fibre-grating sensor. The sensor was applied externally to the rib-cage and interrogated in the lateral (edge) filtering scheme. The study was performed on 34 healthy volunteers. Statistical data analysis of the time lag between the fibre-grating sensor and the reference pneumotachograph was preceded by the removal of the unwanted heartbeat signal by wavelet transform processing.

Main results: The results show a consistent fibre-grating signal advance with respect to the standard pneumatic signal by $(230 \pm 100) \mathrm{ms}$ in both the inspiratory and expiratory phases. We further show that heart activity removal yields a tremendous improvement in sensor accuracy by reducing it from $60 \mathrm{ml}$ to $0.3 \mathrm{ml}$.

Significance: The results indicate that the proposed measurement technique may lead to a more reliable triggering decision. Its imperviousness to air leaks, non-invasiveness, lowcost and ease of implementation offer good prospects for applications in both clinical and homecare ventilation.

Keywords: Optical fibres, Long-period grating sensor, Non-invasive mechanical ventilation, Ventilator triggering 


\section{Introduction}

Mechanical ventilation is one of the most commonly applied techniques in the treatment of patients who cannot match the level of respiratory effort required to maintain a proper breathing cycle. The major driving force behind the increasing use of non-invasive ventilation (NIV) is that it provides ventilator support through the patient's upper airways via a facemask or helmet, which preserves the airway defense mechanisms, reducing patient discomfort and avoiding the complications caused by intubation (Baudouin et al 2002, Mehta and Hill 2001). However, the interface of the mask with the patient inevitably promotes air leaks (Ueno et al 2011) that can increase patient-ventilator asynchrony, and hence cause sleep fragmentation, reduce patient tolerance to NIV and reduce the efficiency of NIV (Hess 2011).

During spontaneous breathing, the stimulus to breathe generated in the central nervous system travels via the phrenic nerve to the diaphragm (figure 1). Contraction of the diaphragm causes the chest-wall and lung expansion that results in generation of airflow (Nava et al 2009, Verbrugghe and Jorens 2011). Ideally, mechanical ventilators should trigger/cycle in synchrony with the stimulus generated by the central nervous system. The best currently available neuro-ventilatory coupling integration for triggering is neurally adjusted ventilatory assist (NAVA), where triggering is accomplished by capturing the electrical activity of the diaphragm by a special catheter placed into the patient's esophagus (Spahija et al 2010, Verbrugghe and Jorens 2011). The estimated time lag between the initiation of inspiration and the associated diaphragm innervation of about $20 \mathrm{~ms}$ in healthy subjects (Verbrugghe and Jorens 2011) indicates the high accuracy of NAVA (Cordioli et al 2013). On the other hand, the invasiveness of NAVA technology is a significant drawback.

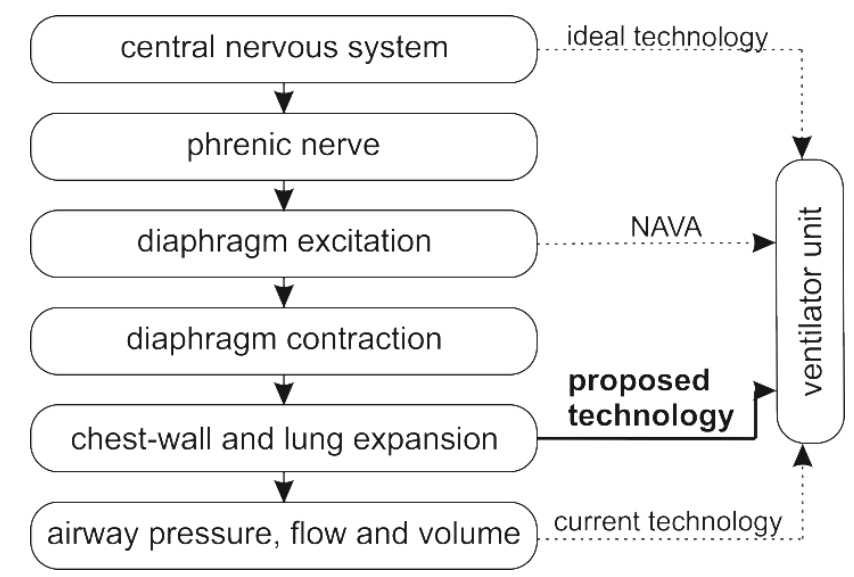

Figure 1. Neuro-ventilatory coupling sequence from Nava et al (2009) and Verbrugghe and Jorens (2011) updated with the measurement scheme proposed here.

Commonly used mechanical ventilators are equipped with non-invasive pneumatic (flow, pressure and/or volume) sensors for detecting the patient's respiratory effort. The sensor triggering mechanism detects inspiration from a drop in pressure or increase in airflow in the ventilatory circuit, which come at the end of the neuro-ventilatory coupling sequence, figure 1. Unlike NAVA, all pneumatic-triggering variables may be affected by air leaks, which play a major role in generating time lag in the feedback to the ventilator. The reported lags of mechanical ventilators applied to patients with pneumatic triggering reach 80-550 ms (Spahija et al 2010 and references therein). The time lag with which the respiratory effort is detected represents the dominant source of the most common patient-ventilator asynchronies and triggering errors (the failure to trigger the ventilator, auto-triggering without patient's effort, delayed triggering, premature cycle or prolonged cycling (Epstein 2011)).

Currently available non-invasive technologies independent of pneumatic variables are based on detection of changes in thoracic impedance (Hummler et al 1996, Nikischin et al 1996), generation of induction 
signal due to the movement of a coil in a magnetic field (Nikischin et al 1996), recording of surface electromyograms (EMG) of the scalene (neck) muscles (Hug et al 2006) and of parasternal intercostal muscle (timing of EMG signal peak similar to the diaphragm signal peak) (Sinderby et al 1996). However, they suffer from drawbacks (prolonged trigger delay, auto-triggering and trigger failure, reliability depending on correct placement, low signal-to-noise ratio, contamination by nonphysiological signals or cross-talk with signals from other muscles) that limit their usage to laboratory studies.

As the fibre-optic technology lends itself to robust, noninvasive and electrically insensitive measurements on humans, efforts have been invested into investigations of non-invasive respiration monitoring using fibre grating based sensors and systems. In a pioneering study by Wehrle et al. (Wehrle et al 2001) fibre Bragg gratings (FBG) were used to measure the dynamic strain of human chest during respiration. This concept was extended, within the EU project OFSETH (Optical Fibre Sensors Embedded into technical Textile for Healthcare monitoring 2007), to the development of wearable sensing technology for monitoring of vital physiological parameters. The sensors developed in that project were based on measuring elongation of the abdominal circumference during respiration (Grillet et al 2008). Besides FBGs, long-period gratings have been used as respiration monitors either as single sensors (Petrovic et al 2014) or a serially multiplexed sensor array (Allsop et al 2007). However, in none of these studies was the triggering potential of the technique under study evaluated with respect to the current clinical standard - pneumotachography. Therefore, there is still a strong need for the identification of a non-invasive technology capable of improving patient-ventilator synchrony in clinical settings.

In an attempt to find a non-invasive triggering mechanism that precedes pneumatic triggering in the neuro-ventilatory coupling sequence, we have developed a new method and apparatus for continuous monitoring of respiration during NIV. The method is based on the measurement of chest-wall motion and relies on an easily-applicable non-invasive optical-fibre sensing technology. The technical solution is based on the use of a single fibre-grating sensor attached to the patient's thorax and a monochromatic interrogation scheme. We have previously shown that the change in curvature of the chest-wall during breathing is linearly correlated with the tidal volume (the volume of air moved into or out of the lungs within each breath) (Petrovic et al 2014). However, for the application of this solution in NIV it is crucial to demonstrate that the lag of the curvature signal is comparable to or smaller than that of the signal generated from the standard airflow sensors. The purpose of this study was to investigate the time relationship between these two signals, and hence test the possibility of using chest-wall motion as a confident triggering mechanism. We paid particular attention to elimination of the heartbeat signal shown to be detrimental for accurate detection of the inhalation and exhalation onsets (Ivanovic and Petrovic 2016).

The paper is structured as follows. In Section 2, the working principle of long-period fibre grating sensors and the monochromatic interrogation scheme for recording fibre-grating curvature signals are described. The measurement protocol and data-analysis method used to determine the time relationship between these and airflow signals are also described therein. The results of measurements on a set of 34 healthy volunteers are analyzed in Section 3. In Section 4, technical limitations and possible improvements of the proposed technology are discussed, and the proposed method is compared with the existing solutions. In section 5, we summarize the conclusions and outline directions of future work.

\section{Methods}

\subsection{LPG curvature sensor}

Changes in torso curvature were measured by a long-period fibre grating (LPG) sensor of bending. The LPG is typically fabricated by using a laser beam to change the refractive index of the fibre core with a spatial period ranging from tens to several hundred micrometers (Erdogan 1997, Kashyap 2010). In these 
gratings, the energy couples from the fundamental core mode to forward-propagating cladding modes at certain resonant wavelengths determined by the phase-matching condition

$$
\lambda=\left(n_{c o}^{e f f}-{ }^{v} n_{c l}^{e f f}\right) \Lambda,
$$

where $n_{c o}^{\text {eff }}$ represents the effective index of the core mode, ${ }^{v} n_{c l}^{\text {eff }}$ the effective index of the $v$ th cladding mode, $\Lambda$ is the period of the grating and $\lambda$ the resonant wavelength. The light in the cladding modes is quickly attenuated, which results in a transmission spectrum consisting of a series of loss bands centered at the resonant wavelengths. Each band corresponds to the coupling of the fundamental mode to a cladding mode of a different order $v$ (James and Tatam 2003, Othonos and Kalli 1999). Beside resonant wavelength, attenuation bands are also characterized with their magnitude, which depends on the modecoupling strength and the grating length, and width, which also depends on the fibre and the grating dispersion.

Modulation of the core and/or cladding guiding properties as a consequence of an external perturbation, such as strain, bending or temperature change, modifies the spectral response of the LPG, which can be utilized for sensing purposes (James and Tatam 2003). Bending, in particular, induces strain in the fibre core and cladding, which results in the refractive index change defined by the fibre-material strain-optic coefficients. The grating cross-sensitivity to the changes in the refractive index of the fibre environment has been effectively suppressed by using a progressive three-layered fibre with an isolating lower-index outer cladding. Encapsulation of the LPG into a silicon rubber, allowed for sufficient reduction in the grating sensitivity to rapid temperature fluctuations as well as an easier sensor manipulation (Petrovic et al 2013 and references therein).

Spectrum alternations due to bending can be observed as changes in position, shape and amplitude of the attenuation bands, allowing for the bend-sensing technique to be based on the measurement of the resonance wavelength shift or the spectral amplitude change at one wavelength. For sensing based on the resonant wavelength shift, measurement of the transmission spectrum is needed which implies the use of a broadband light source and optical spectrum analyzer (OSA). While convenient in the laboratory, this approach is impractical for clinical measurements due to the high cost and time-consuming data acquisition of OSAs.

\subsection{Measurement scheme}

Here, the thorax motion is detected using the monochromatic lateral (edge) filtering interrogation technique which relies on the transmitted power measurement at a specified wavelength, figure 2 . The nearly monochromatic measurement was enabled by using a temperature- and current-stabilized distributed feedback (DFB) laser diode with a bandwidth much smaller than the width of the grating attenuation band. The diode was set to the wavelength of $1470.73 \mathrm{~nm}$. The output power was detected by a photodiode (Petrovic et al 2014). All components of the interrogator are commercially available at lowcost.

The LPG sensitivity (the smallest change in curvature that the sensor can detect) was $0.02 \mathrm{~m}^{-1}$. To set the grating to the optimum working point (WP), we need to tune the grating into the resonance with the laser wavelength by finding the proper curvature (location and orientation) of the sensor on the subject's thorax. 
a)

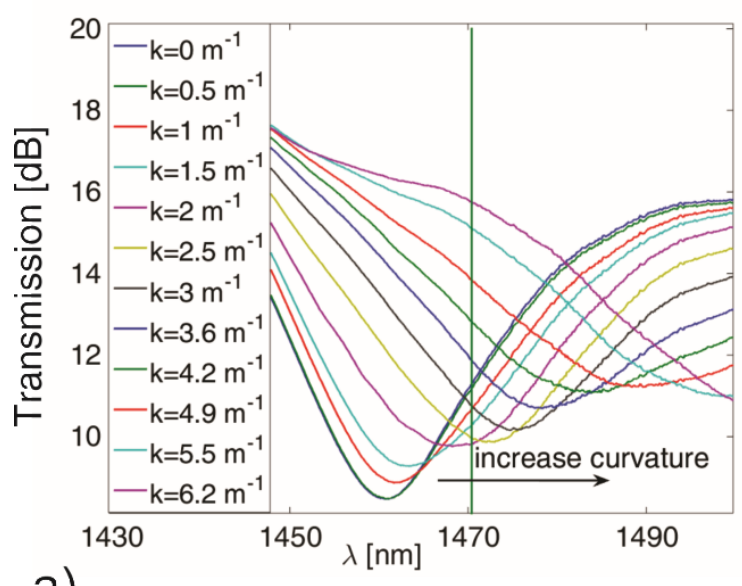

Figure 2. Power sensitivity of the LPG to bending measured by a) OSA (Agilent 86142b), b) sensor power response at $1470.4 \mathrm{~nm}$. Curvatures below the working range cause a nonunique response of the sensor and curvatures above drive the sensor into saturation.

\subsection{Measurement protocol}

During all measurements, subjects were asked to remain in the supine position. The LPG sensor, encapsulated in $8 \mathrm{~cm}$ long silicone rubber, was placed on the thorax stiff area on the right side and fixed below an elastic bandage that goes around the subject's thorax. The stiff area was selected to avoid multiple curvatures along the grating. Measurement on the right side of the thorax was less susceptible to thorax motion caused by the heartbeat. The reference measurements were performed by the pneumotachograph built into a mechanical ventilator Hamilton C3 (Part no. 155362), figure 3. A typical clinical measurement was emulated by placing the pneumotachograph in a tube extension of an oronasal mask and connecting it to the ventilator. The subjects were asked to breathe naturally through an oronasal mask. During the measurements the ventilator was not working in any of its modes. In that manner we assured that the ventilator was not affecting subject's breathing, so that both airflow and thorax expansion were only due to the subject's own respiration effort. Six sets of measurements, $100 \mathrm{~s}$ each, were carried out on every subject.

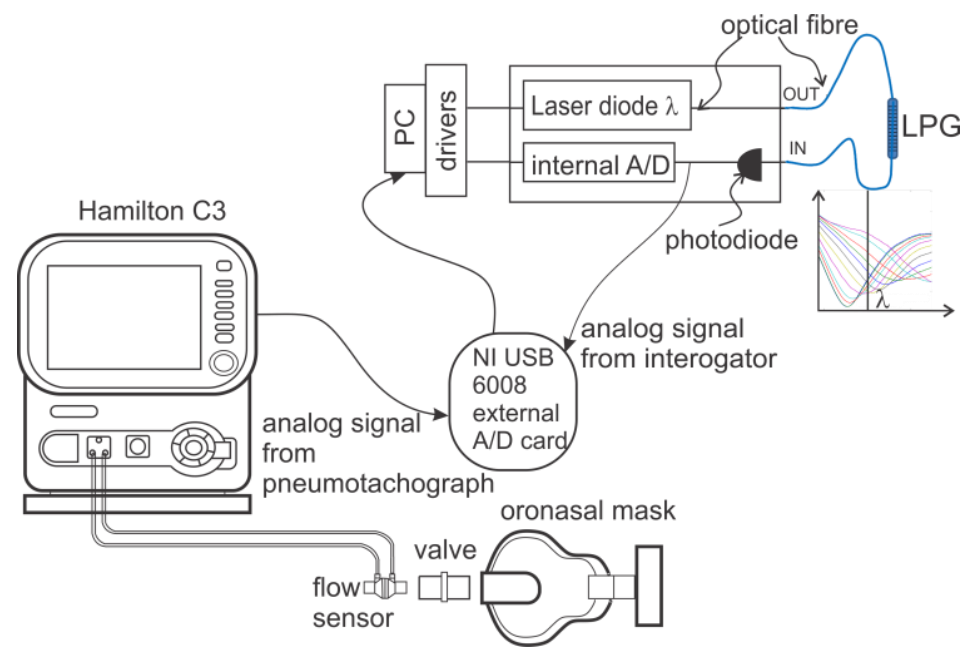

Figure 3. Measurement scheme with the analogue read-out from the fibre-optic interrogator and pneumotachograph. 
To determine the time relationship between the signals generated by the chest-wall motion and airflow, analogue electronic signals from the LPG sensor and pneumotachograph were directly fed to the same external analogue-to-digital converter (NI USB6008). A LabVIEW program (http://www.ni.com/labview) was developed for data acquisition and raw signal storage. Signals were processed off-line by MATLAB (https://www.mathworks.com/products/matlab.html). Time resolution of the measurement was limited by the $100 \mathrm{~Hz}$ sampling frequency.

Following the approval of the study protocol by the ethics committee of the School of Medicine, University of Belgrade, 34 healthy volunteers (19 females and 15 males) with normal pulmonary function and no evidence of chest-wall abnormalities were included into the study. The study was carried out at the University Clinical Hospital Zemun, Serbia. The volunteers were recruited among hospital staff and employees of Vinca Institute, aged $37 \pm 11$ years and with BMI $24.3 \pm 5.5 \mathrm{~kg} \mathrm{~m}^{-2}$, where the first number represents the mean value and the second the standard deviation of the set. They were chosen to render a BMI distribution covering the cases of BMI lower than 19.5 and higher than 25, at which we previously observed large deviations in the LPG sensor signal timing (Ivanovic et al 2015).

\subsection{Data analysis}

At the beginning of the first measurement, every subject was asked to hold her or his breath for about $10 \mathrm{~s}$ and this interval was used to determine the zero-level of the airflow signal. The volume signal from the pneumotachograph was calculated by integrating the flow signal after subtraction of the zero-level. Upon the integration of the flow signal, a baseline drift in the pneumotachograph volume signal appears. The drift was eliminated by subtracting the baseline signal calculated by piece-wise polynomial fitting with the window size of approximately 5 respiratory cycles. The LPG volume signal was obtained by the calibration procedure established in Petrovic et al 2014. Even though the drift of the LPG power signal of around $70 \mathrm{ml} / \mathrm{min}$ (less than $5 \mathrm{ml} /$ breath) on average was negligible, the same procedure of drift elimination was performed on the LPG signals.

The signal generated by heart activity superposes on the chest-wall-motion signal and in our measurements it is an unwanted part of the signal (Ivanovic and Petrovic 2016). The wavelet filtering method was applied on the drift-less LPG signals to eliminate the heart signal from the respiratory activity, figure 4. This method was applied in the following steps: (i) the continuous Morlet-wavelet transform of the signal, (ii) scale-dependent thresholding of the wavelet coefficients and (iii) inverse wavelet transform to recover the respiratory signal uncontaminated by the heart signal. The time-scale representations (e.g. scalogram in figure $4 b$ ) of the signals were plotted for a selection of the pseudofrequency threshold values that separates the respiratory and heart activity. The threshold was manually selected by visual inspection of the scalogram and by applying the established knowledge of respiratory and heart activity frequency bands. The filtered respiratory activity was obtained by setting the wavelet coefficients above the pseudo-frequency threshold to zero prior to carrying out the inverse wavelet transform. Similarly and only for the purpose of plotting figure 4, the heart activity signal was extracted by zeroing below-threshold coefficients and performing the inverse wavelet transform. A global threshold value corresponding to the pseudo-frequency of $0.8 \mathrm{~Hz}$ was selected for processing data of all subjects. The chosen value was slightly below the lower boundary of the normal cardiac frequency range in resting adults (50 beats per minute). The additional requirement for accepting this global threshold was that the frequency corresponding to the peak power spectral density within physiologically plausible cardiac frequency range (30-220 beats per minute) was in all subjects higher than the pre-selected global threshold. [Addison and Watson 2004]. 

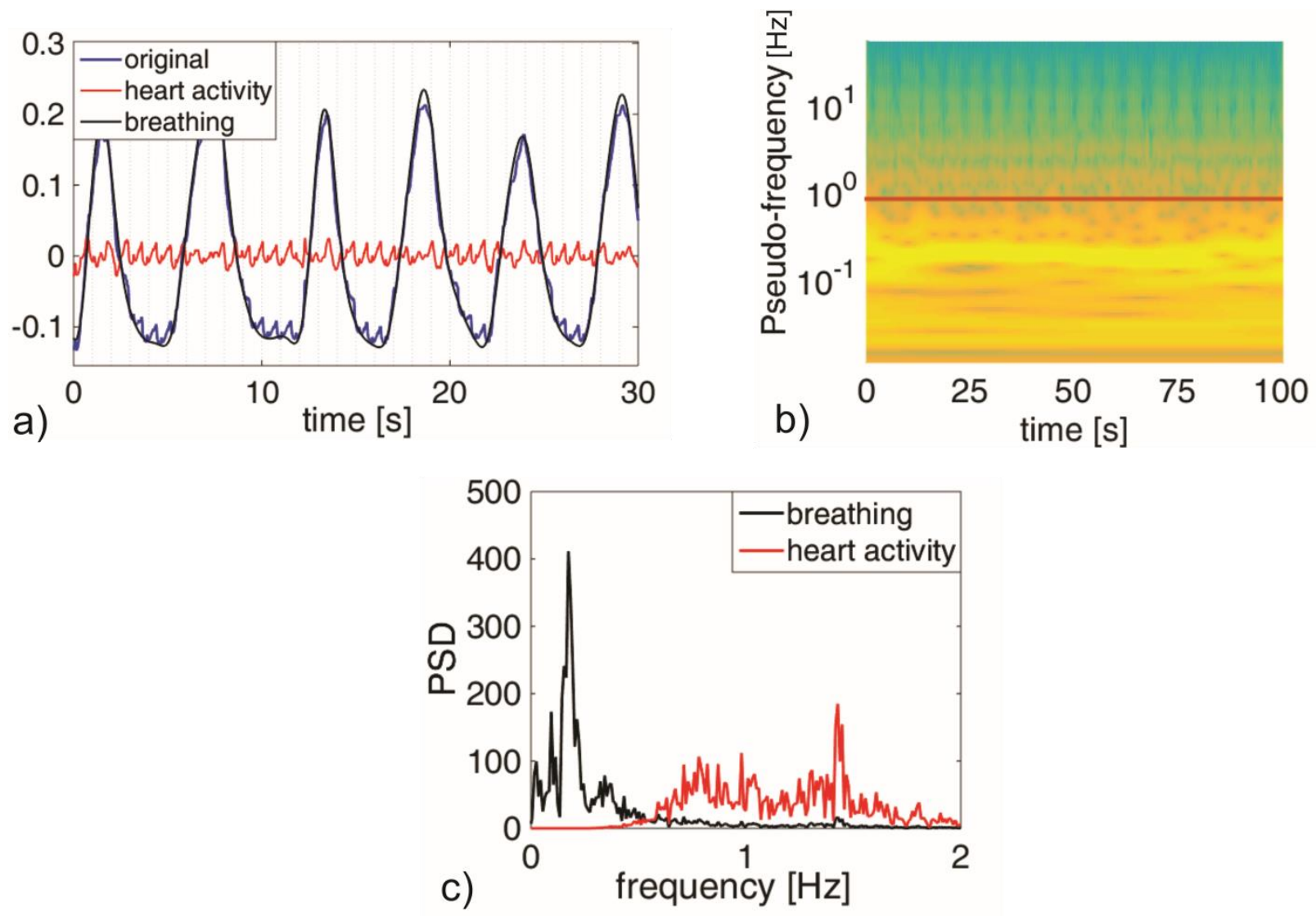

Figure 4 a) Original LPG signal (blue line) with separated respiratory (black line) and heart activity (red line) components, b) scalogram with the scale-threshold line at $0.8 \mathrm{~Hz}$; c) frequency content (power spectrum density (PSD)) of respiratory and heart activity components of the signal.

We further defined two parameters for the sensor delay performance metric: an average time shift at which the cross-correlation of the LPG and pneumotachograph volume signals reaches maximum over the whole inspiratory/expiratory sequence, $\mathrm{d}_{\mathrm{xcorr}}$, and the same measure performed only on the first $10 \%$ of the inspiratory/expiratory amplitude over the whole sequence, $d_{10 \%}$ (figure 5 ). The purpose of the latter metric was to determine the time relationship in the part of the signal important for triggering/cycling. All time intervals were expressed as delays of the LPG signal with respect to the pneumotachogram, the negative sign of the delay meaning that the LPG signal precedes the pneumotachograph signal. 


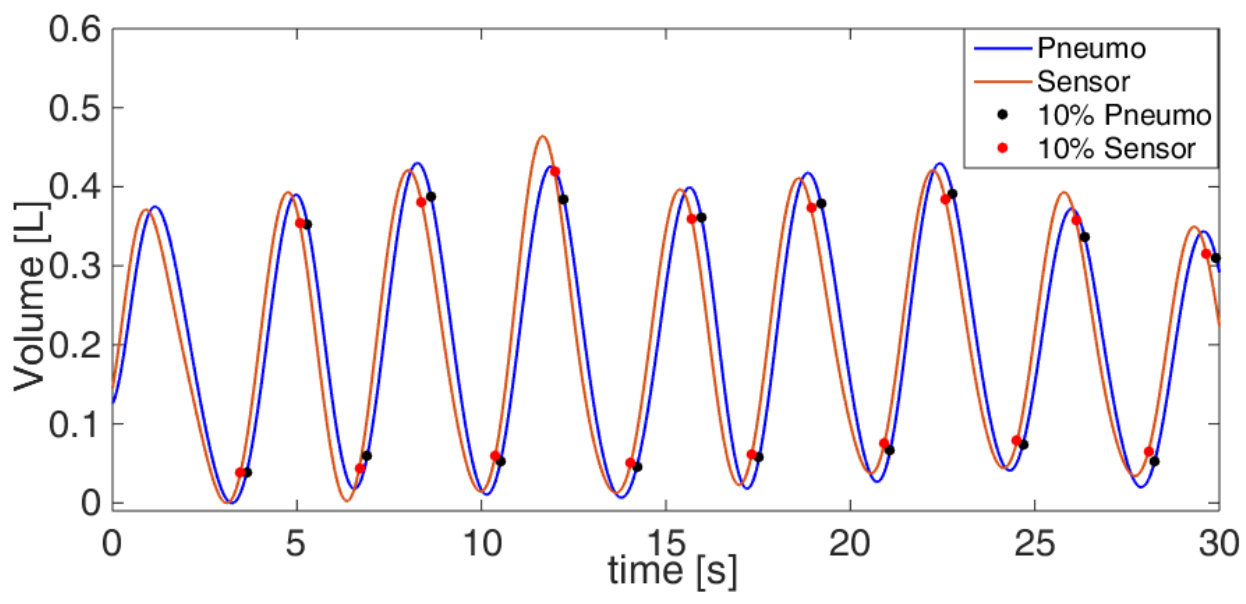

Figure 5. Pieces of typical LPG and pneumotachograph signals with marked moments at which each signal reaches $10 \%$ of its amplitude in the inspiratory and expiratory phases.

\section{Results}

The results obtained on the whole set are shown in numbers in table 1 and graphically in figure 6 . The results show that the chest-wall-motion signal measured by the LPG sensor advances with respect to the volume signal measured by pneumotachograph by about $230 \mathrm{~ms}$ on average in both the inspiration and expiration phases. The standard deviations of around $50 \%$ in all defined measures are presumably due to a large variation in the chest-wall motion in different people during breathing and the different location and orientation of the sensor on the subject's thorax.

Table 1. Statistical data for the whole set.

\begin{tabular}{|ccccc|}
\hline \multirow{3}{*}{ Delay } & \multicolumn{3}{c|}{$\begin{array}{c}\text { Different metrics } \\
\text { for delays [ms] }\end{array}$} \\
\cline { 2 - 5 } & \multicolumn{2}{c|}{$\begin{array}{c}\text { Inspiratory } \\
\text { phase }\end{array}$} & \multicolumn{2}{c|}{$\begin{array}{c}\text { Expiratory } \\
\text { phase }\end{array}$} \\
\cline { 2 - 5 } & $\mathrm{d}_{\mathrm{xcorr}}$ & $\mathrm{d}_{10 \%}$ & $\mathrm{~d}_{\mathrm{xcorr}}$ & $\mathrm{d}_{10 \%}$ \\
\hline mean & -230 & -220 & -230 & -240 \\
std & 100 & 120 & 100 & 130 \\
\hline
\end{tabular}



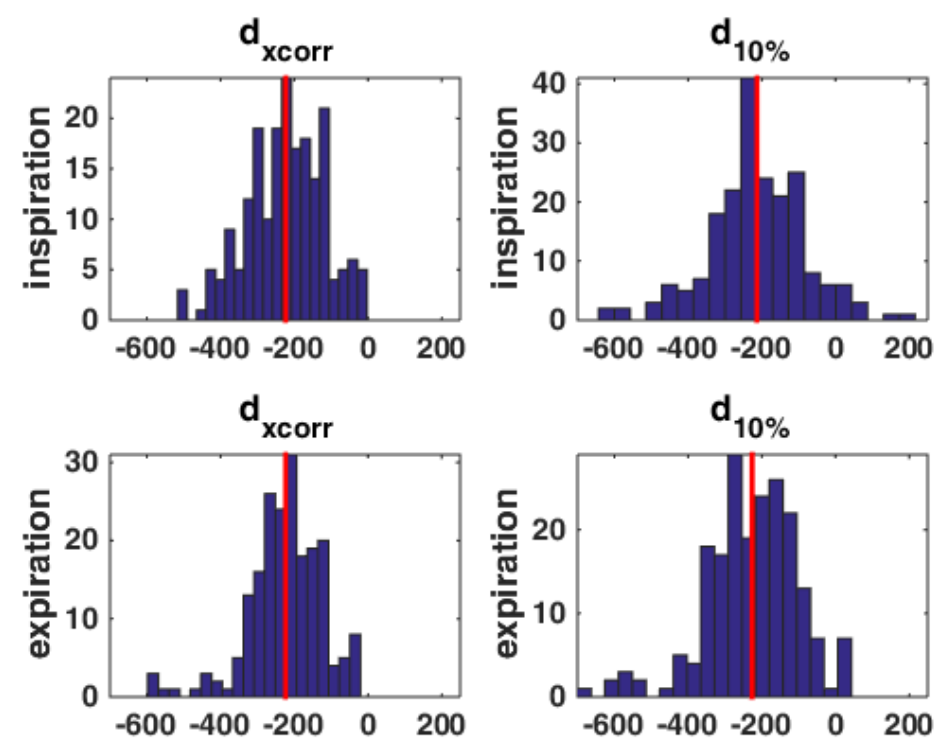

Figure 6. Distribution of delays of LPG sensor signals with respect to pneumotachograph for different metrics. Red lines show the mean values.

The measurement error largely depends on the accuracy of finding extrema of the respiratory signal. It can be severely aggravated by the heart activity, the amplitude of which is comparable to the changes in respiratory signal in the end-inspiratory and end-expiratory phases. Consistent positioning of the sensor to the right side of the rib cage can significantly reduce the amplitude of the heart activity component. However, in some subjects it could still reach $60 \mathrm{ml}$, constraining the sensor accuracy. Elimination of the heart activity signal using the continuous wavelet transform led to improvement of the sensor accuracy to $0.3 \mathrm{ml}$, figure $4 \mathrm{a}$. Furthermore, this led to a more precise determination of the signal minima and maxima, and hence to the reduction in the standard deviation of the time delay recorded for each subject (figure 7). Although the wavelet filtering method was applied offline, the results show clear separation between the respiratory and heart activity in all tested subjects with a ubiquitous threshold value, which provides promising evidence of the feasibility of automated application.

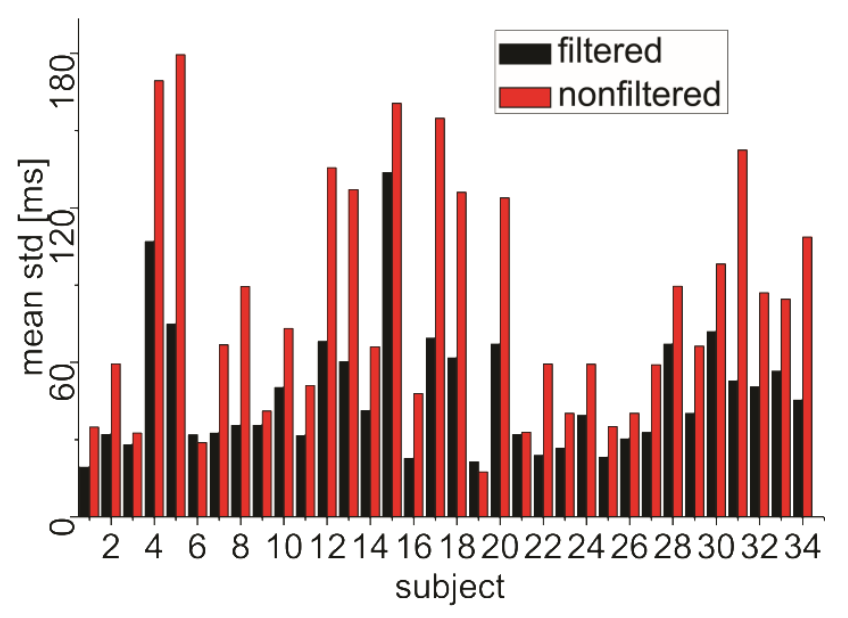

Figure 7. Average standard deviation of the time delay calculated for the whole inspiratory phase for each subject (black- without heart-beat signal, red - with heart-beat signal). 


\section{Discussion}

Other investigators have tested the possibilities of using different sensors and triggering methods. In table 2, we compare the technique proposed here with techniques reported in the literature in terms of the sensor signal timing, cross-talk with patient movement and the stage of development. We use the airflow sensor as the common reference and concentrate on non-invasive techniques. While in this study, we do not address the issue of trigger delay (which depends on the ventilator settings and processing algorithm that vary from ventilator to ventilator), for completeness, we list the results of others for triggers that use different sensing techniques.

Table 2. Comparison with available data.

\begin{tabular}{|c|c|c|c|c|c|c|}
\hline method & $\begin{array}{l}\text { signal delay } \\
\text { with respect } \\
\text { to flow } \\
\text { signal }[\mathrm{ms}]\end{array}$ & $\begin{array}{l}\text { trigger } \\
\text { delay } \\
{[\mathrm{ms}]}\end{array}$ & $\begin{array}{l}\text { Invasive } \\
\text { ness }\end{array}$ & $\begin{array}{l}\text { cross-talk } \\
\text { with } \\
\text { patient } \\
\text { moves }\end{array}$ & usage & comment \\
\hline NAVA & $X$ & 100 & YES & NO & clinical & $\begin{array}{c}\text { Study Spahija et al } \\
\text { (2010) on intubated } \\
\text { intensive care unit } \\
\text { patients }\end{array}$ \\
\hline $\begin{array}{c}\text { pneumatic } \\
\text { measurement }\end{array}$ & 0 & $80-550$ & NO & NO & clinical & $\begin{array}{l}\text { Studies Nikischin et } \\
\text { al (1996), Spahija et } \\
\text { al (2010) and the } \\
\text { references therein on } \\
\text { pressure support } \\
\text { ventilated patients }\end{array}$ \\
\hline $\begin{array}{l}\text { LPG chest- } \\
\text { wall curvature } \\
\text { measurement }\end{array}$ & -230 & $X$ & NO & YES & laboratory & $\begin{array}{l}\text { study on healthy } \\
\text { vounteers }\end{array}$ \\
\hline $\begin{array}{l}\text { impedance } \\
\text { measurement }\end{array}$ & 44 & 170 & NO & YES & laboratory & $\begin{array}{l}\text { Studies Hummler et } \\
\text { al (1996), Nikischin } \\
\text { et al (1996) on } \\
\text { infants }\end{array}$ \\
\hline $\begin{array}{c}\text { induction } \\
\text { measurement }\end{array}$ & -13.5 & 90 & NO & YES & laboratory & $\begin{array}{l}\text { Study Nikischin et al } \\
\text { (1996) on infants }\end{array}$ \\
\hline
\end{tabular}

Two studies performed on infants (Hummler et al 1996, Nikischin et al 1996) that compare flow- and impedance-triggered systems show that chest-wall signals detected by changes in thoracic impedance have longer sensor and trigger delays with less consistent response time in comparison with flow signals. Moreover, the impedance-triggered system is highly susceptible to false triggering, which makes this system unreliable for mechanical ventilation synchronization. In the study by Nikischin et al (1996), the flow- and impedance-triggered systems were additionally compared to an induction (magnetic-coil) system that records abdominal motion. This study reported that the induction signal was registered 13.5 ms on average before the airflow signal when measured on infants. These studies were performed only on 
infants, and therefore we could not use their results for direct comparison of impedance, induction and curvature methods. To the best of the authors' knowledge, impedance- and induction-triggered systems have not been used in adult patients, and the techniques developed for continuous monitoring of breathing (respiratory inductance plethysmography (Watson 1980), fibre-optic respiratory plethysmography (Allsop et al 2007, Davis et al 1997, Drummond et al 1996), optoelectronic plethysmography (Aliverti et al 2001)) and for determination of lung impedance (Kaczka et al 1999) have not been used for the triggering purpose. In the study by Wehrle et al. (2001), the authors reported that fibre Bragg grating (FBG) sensor can be used for detecting deformation of the thorax movement of adults and for triggering a pulse burst of electrically assisted ventilation when one lung is to be excited in phase with the other working lung. As opposed to focusing on a particular health problem, in the study presented here, we investigated the noninvasive triggering mechanism that could precede pneumatic triggering in the neuro-ventilatory coupling sequence. Since the time lag with which the respiratory effort is detected represents the dominant source of the most common patient-ventilator asynchronies, authors believe that the large demonstrated advance by more than $200 \mathrm{~ms}$ of the LPG with respect to all other reported sensors can lead to more confident triggering decisions.

In the current realization of the LPG interrogation scheme, the laser tuning range of $5 \mathrm{~nm}$ was not wide enough to set the laser wavelength to the optimal WP for every subject (figure 2b). Instead, the grating was tuned into the resonance with the laser by finding the curvature on a subject's thorax that corresponds to the WP, thus making the optimal sensor position subject dependent. The WP was sought among the positions far from the ends and the minimum of the grating bend (figure $2 b$ ) in order to avoid saturation and non-unique response of the sensor, respectively. It was typically found in a few iterations. This procedure of finding an optimal WP of the sensor is imposed by a technical and not fundamental limitation. The second version of the interrogation scheme will use a broadband laser diode, which will allow for automatic finding of the sensor WP regardless of its orientation on a subject.

Finally, an alternative solution is to use fibre Bragg gratings (FBGs). However, LPGs have higher sensitivity to bending since the cladding modes generated by an LPG are more susceptible to the bendinduced refractive-index changes across the fibre than the core-confined back-propagating modes generated by an FBG.

\section{Conclusion}

The research presented in this paper tested the feasibility of using an optical LPG fibre sensor to provide a reliable non-invasive feedback to the ventilators used in NIV. The changes in chest-wall curvature registered by single LPG sensor were measured simultaneously with the air-flow through an oronasal mask with a pneumotachograph. Our data set of the volume signals shows that the chest-wall-motion signal is advanced with respect to the airflow signal by about $230 \mathrm{~ms}$, indicating that the LPG scheme, alone or in conjunction with pneumatic, may be used to reduce patient-ventilatory asynchrony. In addition, the demonstrated technique is not affected by air leaks with which patient-ventilator asynchrony is commonly associated. It is non-invasive, simple, cost-effective, easy to implement and can be used on both male and female patients.

The presented results provide a proof-of-principle that the proposed method is capable of monitoring breathing in real-time with an advantage of earlier detection than a standard airflow sensor. Further comprehensive clinical studies will be performed to establish the application range of LPG sensors with regard to different types of illnesses and obstructions, in particular to COPD (chronic obstructive pulmonary disease). Within these studies, other methods (diaphragmatic EMG or transdiaphragmatic pressure) will be used to determine the limitations of the LPG response to the muscular activity and identify nonresponders. Finally, particular attention will be paid to the elimination of errors that stem from the sensor sensitivity to the body movements not associated with breathing. 


\section{Acknowledgments}

Authors acknowledge financial support from the Ministry of Education, Science and Technological Development of Serbia (Project No. III45010) and European Union's Horizon 2020 research and innovation programme under the Marie Sklodowska-Curie grant agreement No. 691051, and thank volunteers and University Clinical Hospital Zemun, Serbia for logistic support of the study.

\section{References}

Addison P and Watson J 2004 Secondary transform decoupling of shifted nonstationary signal modulation components: application to photoplethysmography Int J Wavelets Multiresolut Inf Process 2 43-57

Aliverti A, Dellaca R, Pelosi P, Chiumello D, Gattinoni L and Pedotti A 2001 Compartmental analysis of breathing in the supine and prone positions by optoelectronic plethysmography Ann Biomed Eng 29 60-70

Allsop T, Carroll K, Lloyd G, Webb DJ, Miller M and Bennion I 2007 Application of long-period-grating sensors to respiratory plethysmography J Biomed Opt 12(6) 064003 (pp13)

Baudouin S, Blumenthal S, Cooper B, Davidson C, Davison A, Elliott M, Kinnear W, Paton R, Sawicka E and Turner L 2002 Non-invasive ventilation in acute respiratory failure Thorax 57 192-211

Cordioli R L, Akoumianaki E and Brochard L 2013 Nonconventional ventilation techniques Curr Opin Crit Care 19(1) 31-7

Davis C, Mazzolini A and Murphy D 1997 A new fibre optic sensor for respiratory monitoring Australas Phys Eng Sci Med 20(4) 214-19

Drummond G B, Nimmo A F and Elton R A 1996 Thoracic impedance used for measuring chest wall movement in postoperative patients Br J Anaest 77(3) 327-32

Epstein S K 2011 How often does patient-ventilator asynchrony occur and what are the consequences? Respir Care 56(1) 25-38

Erdogan T 1997 Cladding-mode resonances in short- and long-period fiber grating filters J Opt Soc Am A 14 1760-73

Grillet A, Kinet D, Witt J, Schukar M, Krebber K, Pirotte F and Depre A 2008 Optical fiber sensors embedded into medical textiles for healthcare monitoring IEEE Sensors J 8(7) 1215-22

Hess D R 2011 Patient-ventilator interaction during noninvasive ventilation Respir Care 56(2) 153-65

Hug F, Raux M, Prella M, Morelot-Panzini C, Straus C and Similowski T 2006 Optimized analysis of surface electromyograms of the scalenes during quiet breathing in humans Respir Physiol Neurobiol 150(1) 75-81

Hummler H D, Gerhardt T, Gonzalez A, Bolivar J, Claure N, Everett R and Bancalari E 1996 Patienttriggered ventilation in neonates: comparison of a flow-and an impedance-triggered system Am J Respir Crit Care Med 154 1049-54

Ivanovic M D et al 2015 Rig-cage-movement measurements as a potential new trigger signal in noninvasive mechanical ventilation In Conf Proc IEEE Eng Med Biol Soc. 2015 4511-4

Ivanovic M D and Petrovic J 2016 A long-period fibre grating monitor of respiratory volumes for the use in non-invasive mechanical ventilation Opt Quant Electron 48346 (10pp)

James S W and Tatam R P 2003 Optical fibre long-period grating sensors: characteristics and application Meas Sci Technol 14 R49-61

Kaczka D W, Ingenito E P and Lutchen K R 1999 Technique to determine inspiratory impedance during mechanical ventilation: implications for flow limited patients Ann Biomed Eng 27 340-55

Kashyap R 2010 Fiber Bragg gratings (San Diego, Academic Press)

Mehta S and Hill N S 2001 Noninvasive ventilation Am J Respir Crit Care Med 163 540-77

Nava S, Carlucci A and Ceriana P 2009 Patient-ventilator interaction during noninvasive ventilation: practical assessment and theoretical basis Breathe 5(4) 323-33 
Nikischin W, Gerhardt T, Everett R, Gonzalez A, Hummier H and Bancalari E 1996 Patient-triggered ventilation: a comparison of tidal volume and chestwall and abdominal motion as trigger signals Pediatr Pulm 22 28-34

Optical Fibre Sensors Embedded into technical Textile for Healthcare monitoring 2007, accessed 15 February 2018, <http://www.ofseth.org/>

Othonos A and Kalli K 1999 Fibre Bragg gratings:fundamentals and applications in telecommunications and sensing (Boston, Artech House)

Petrovic M D et al 2013 Fibre-grating sensors for the measurement of physiological pulsations Phys Scr T154 $014022(4 \mathrm{pp})$

Petrovic M D, Petrovic J, Danicic A, Vukcevic M, Bojovic B, Hadzievski Lj, Allsop T, Lloyd G and Webb D J 2014 Non-invasive respiratory monitoring using long-period fiber grating sensors Biomed Opt Express 5(4) 1136-44

Sinderby C, Friberg S, Comtois N and Grassino A 1996 Chest wall muscle cross talk in canine costal diaphragm electromyogram J Appl Physiol 81(5) 2312-27

Spahija J, de Marchie M, Albert M, Bellemare P, Delisle S, Beck J and Sinderby C 2010 Patientventilator interaction during pressure support ventilation and neurally adjusted ventilatory assist Crit Care Med 38(2) 518-26

Ueno Y, Nakinishi N, Oto J, Imanaka H and Nishimura M 2011 A bench study of the effects of leak on ventilator performance during noninvasive ventilation Respir Care 56(11) 1758-64

Verbrugghe W and Jorens P G 2011 Neurally adjusted ventilatory assist: a ventilation tool or a ventilation toy? Respir Care 56(3) 327-35

Watson H 1980 The technology of respiratory inductance plethysmography ISAM Proc $3^{\text {rd }}$ Intl Symp Ambulatory Monitoring (Academic, San Diego) p537

Wehrle G, Nohama P, Kalinowski H J, Torres P I and Valente L C G 2001 A fibre optic Bragg grating strain sensor for monitoring ventilator movements Meas Sci Technol 12 805-9 